\title{
La laïcité américaine aujourd'hui ou la bataille du Premier Amendement
}

\section{Blandine Chelini-Pont et Gregory Mose}

\section{Q OpenEdition}

\section{Journals}

Édition électronique

URL : https://journals.openedition.org/rdr/704

DOI : $10.4000 /$ rdr.704

ISSN : 2534-7462

Éditeur

Presses universitaires de Strasbourg

\section{Édition imprimée}

Date de publication : 31 octobre 2017

Pagination : 83-103

ISBN : 978-2-86820-974-0

ISSN : 2493-8637

Référence électronique

Blandine Chelini-Pont et Gregory Mose, « La laïcité américaine aujourd'hui ou la bataille du Premier Amendement », Revue du droit des religions [En ligne], 4 | 2017, mis en ligne le 15 janvier 2020, consulté le 23 mai 2022. URL : http://journals.openedition.org/rdr/704 ; DOI : https://doi.org/10.4000/rdr.704

\section{(c) (†) \&)}

La revue du droit des religions est mise à disposition selon les termes de la Creative Commons Attribution - Pas d'Utilisation Commerciale 4.0 International - CC BY-NC 4.0. 


\section{LA LÄ̈CITÉ AMÉRICAINE AUJOURD'HUI OU LA BATAILLE DU PREMIER AMENDEMENT}

\section{Blandine CHELINI-PONT et Gregory MOSE}

Université d'Aix-Marseille

\section{RÉSUMÉ}

Les deux clauses du Premier Amendement de la Constitution des États-Unis ont servi à construire une doctrine de la séparation et de la liberté religieuse dont la mise en place législative et judiciaire a été sinueuse jusqu'aux années 1970. L'ensemble a donné naissance à une forme spécifique de laïcité dont l'équilibre apparent a été à nouveau remis en cause depuis les années 1980. La polarisation politique du pays a atteint l'interprétation des clauses en la liant à la défense antagoniste de l'éthos chrétien ou libéral de l'Amérique. La liberté religieuse balance entre clause d'exemption de conscience ou choix de croire. À travers la description de cette « bataille », c'est bien l'âme de l'Amérique et son dosage, entre identité chrétienne et libérale, qui sont en jeu.

\section{ABstract}

The Religion Clauses of the First Amendment to the Constitution of the United States gave birth to a doctrine of church-state separation and a model of religious freedom whose legislative and judicial implementation was convoluted until the 1970s. A unique vision of secularism emerged from decades of uncertainty, but the resulting equilibrium has once more been called into question since the 1980s. The polarization characterizing the country's politics during this period has extended to debates over the interpretation of the Religion Clauses, resulting in a defensive judicial climate in which interpretive stances align either with a conservative Christian or a progressive liberal ethos. Religious freedom swings between conscience-based exemptions and the choice to believe. At stake in this "battle" is the soul of America and its precarious balance between its Christian and liberal identity. 
$\mathrm{D}$ ans l'histoire des États-Unis, le Premier Amendement, qui fonde à la fois le principe de non-établissement et celui de liberté de culte, est le socle à partir duquel s'est constitué ce que l'on peut appeler la « laïcité américaine $^{1}$ », régime de séparation similaire à celui de la France contemporaine $^{2}$, mais d'intention philo-cléricale, pour reprendre l'expression de Denis Lacorne ${ }^{3}$. Le caractère évolutif de son interprétation et de sa compréhension sociale explique les méandres de sa mise en place et ceux de la situation actuelle, complexe et tendue où neutralité de l'État et liberté religieuse sont devenues des objets de la lutte politique. Nous allons dans cette présentation, parcourir rapidement les grandes périodes de la laïcité américaine, en essayant de montrer les étapes qui en ont enrichi le sens et qui permettent de comprendre la tension actuelle entre deux tendances antagonistes et concurrentes.

Nous verrons comment le $\mathrm{XIX}^{\mathrm{e}}$ siècle et le $\mathrm{XX}^{\mathrm{e}}$ siècle jusqu'aux années 1970 ont été occupés par un double questionnement sur les clauses du Premier Amendement (1). Le premier s'est construit autour de la position de l'État américain vis-à-vis de la clause de non-établissement d'une religion, position qui s'est difficilement imposée comme étant strictement séparée et nécessairement neutre, au sens français du terme. Le deuxième questionnement, tout aussi intense, s'est construit autour de l'extension pratique de la clause de libre exercice des cultes, hors du premier cercle des dénominations protestantes « légitimes », en réaction au sort des minorités religieuses et des «petits» cultes $^{4}$. Elle a donné naissance ou a permis de concourir à la construction signifiante quoique également tardive de la liberté religieuse comme mère des libertés américaines ${ }^{5}$.

1. Terme assumé par Élisabeth ZOLLER, dans l'introduction du collectif qu'elle a dirigé, La conception américaine de la laïcité, Paris, Dalloz, 2005. V. également Rigal-Cellard B., "Comment le Premier Amendement de la Constitution américaine définit la laïcité outreAtlantique », Politeia, n 29, 2016, p. 243-252.

2. ChÉLINI-Pont B., "Laïcité française et américaine en miroir», in Quel avenir pour la laïcité cent ans après la loi de 1905, CRDF, $\mathrm{n}^{\circ}$ 4, 2005, p. 107-118. - BARB A., "La laïcité en France et aux États-Unis : perspectives historiques et enjeux contemporains ", Questions internationales, $\mathrm{n}^{\circ} 76,2015$, p. 87-94.

3. LaCorne D., «La séparation de l'Église et de l'État aux États-Unis, les paradoxes d'une laïcité philo-cléricale ", Le Débat, n 127, 2003, p. 63-79. - BARB A., "Une laïcité ouverte aux religions? Le modèle américain », Études, n 4223, 2016, p. 19-34.

4. Issaoui N., Les mouvements religieux minoritaires à l'épreuve du droit états-unien, Aix-enProvence, PUAM, 2017.

5. Durham W. C. et Kirkham D. M., «États-Unis », in Messner F. (dir.), Dictionnaire Droit des Religions, Paris, CNRS Éd., 2010, p. 290-297. - MCCAUlifF C., « Religion and the Secular State in the United States? », in Martinez Torron J. et Durham W. C. (eds), Religion and the 
Cette première phase de mise en place, assez longue dans sa maturation, a cédé le pas à peine stabilisée. Elle a été remplacée par une période autrement polarisée depuis les années 1970, dans laquelle le Premier Amendement est devenu un objet de clivage politique (2). Nous verrons comment la question de la neutralité de l'État a été remise en question depuis maintenant une quarantaine d'années au profit d'une réaffirmation forte de l'éthos chrétien des institutions, y compris dans la pratique du non-établissement. La liberté religieuse de son côté s'est révélée un thème de mobilisation politique majeur, en ce qu'elle aurait été mise en danger par l'État et son « sécularisme » idéologique. Les décennies 1990 et 2000 ont vu se multiplier des lois fédérales et des contentieux judiciaires fondés sur la défense de la liberté religieuse, indûment négligée ou subordonnée à des impératifs publics abusifs ${ }^{6}$.

Notre dernière partie, portant sur la période contemporaine, s'attachera à mesurer comment la polarisation commencée dans les années 1970 s'est accentuée (3). Le débat sur la liberté religieuse menacée par les empiétements de l'État s'est déplacé subrepticement et a donné naissance à l'exigence d'un droit d'exemption religieuse pour les personnes et les entreprises, quand lois et jurisprudence fédérales portent atteinte - selon la position des défenseurs de cette thèse - à l'éthique sexuelle et reproductive des croyants. Ce glissement sera-t-il temporaire face aux recompositions visibles de cette société dans son rapport au religieux, à la religion et au politique? Rien n'est moins sûr.

\section{LA FABRICATION D'UNE DOCTRINE DU PREMIER AMENDEMENT (1788-1960)}

Beaucoup a été écrit sur la solution extraordinairement «simple» qui a été mise par écrit dans le Premier Amendement du Bill of Rights (Déclaration des droits) de la Constitution fédérale. La formule choisie «Congress shall make no law respecting an establishment of religion, or prohibiting the free exercise thereof » n'a jamais empêché les débats sur sa vraie signification, et ce, dès le commencement. Chacun connaît le commentaire donné par Thomas Jefferson dans sa lettre collective aux pasteurs baptistes du

Secular State. National Reports (XVIIIth Congress of the International Academy of Comparative Law), Madrid, Facultad de Derecho de la Universidad Complutense, 2015, p. 793-812.

6. BARB A., Les dilemmes de l'État laïque : les politiques d'accommodements religieux aux États-Unis des années 1960 à nos jours, Aix-en-Provence, PUAM, 2017. 
Connecticut en janvier 1802 (Danbury Letter). Dans ce texte bref, Jefferson insiste sur le caractère sacré de la conscience et de ses droits («religion is a matter which lies solely between Man and his God, that he owes account to none other for his faith or his worship »), pour expliquer que c'est précisément en vue de protéger les droits de la conscience que la législature du Congrès a construit un mur de séparation entre l'Église et l'État. Mais la question restait ouverte de savoir si la séparation avait comme but de protéger les citoyens d'une religion étatique, de protéger l'État d'une religion en particulier ou de la religion en général, ou de protéger en priorité la conscience libre des citoyens. Et cette question correspond à ce que nous définirions comme le caractère plastique du Premier Amendement américain, évoluant au fur et à mesure des contextes nouveaux, des conflits et aujourd'hui des enjeux politiques qui s'y mêlent.

\subsection{DU NON-ÉTABLISSEMENT À LA NEUTRALITÉ DE L'ÉTAT}

Dans les premières décennies qui ont suivi l'adoption de la Constitution fédérale, les nouveaux États fédérés se sont divisés sur l'applicabilité du Bill of Rights fédéral dans leur propre système constitutionnel. La plupart ont ratifié les dix amendements. Ils ont admis la liberté de conscience et de culte et proclamé le désétablissement, sans que tous les États ne l'adoptent cependant, puisque la Géorgie (1798), le Connecticut (1818) et le Massachusetts (1833) ont gardé leurs Églises établies et ont pu maintenir des interdictions de culte, notamment pour les catholiques, jusqu'à ces mêmes dates.

Cependant, la compréhension que les États fédérés ont eue de leur « séparation » réelle a été extrêmement variable pour ne pas dire relative jusqu'à la fin du $\mathrm{XIX}^{\mathrm{e}}$ siècle et encore au $\mathrm{Xx}^{\mathrm{e}}$ siècle. Il allait par exemple de soi que les écoles publiques enseignent la religion calviniste dans la Bible du roi Jacques. L'une des grandes batailles des diocèses catholiques a été à cette période de mettre sur pied des écoles paroissiales gratuites, non sans avoir dénoncé la partialité confessionnelle des pouvoirs publics. C'est autour de l'école publique et de son lien au protestantisme que la plupart des contentieux se portent alors, qui vont forcer la Cour suprême fédérale à formuler progressivement une doctrine de neutralité. Dans l'arrêt célèbre, Everson v. Board of Education (330 US 1, 194777), qui décrète par ailleurs l'application

7. Un contribuable de la commune d'Ewing dans le New Jersey avait fait un recours contre l'extension du transport des bus scolaires aux écoles paroissiales catholiques. Il estimait que cette extension était une violation de la Constitution du New Jersey; ayant perdu 
de la clause fédérale du non-établissement à tous les États selon le procédé dit d'incorporation, le principe est tardivement posé que:

«La clause de non-établissement du Premier Amendement signifie au moins ceci : aucun État non plus que le gouvernement fédéral ne peut mettre en place une Église. Ni passer des lois qui aident une religion, aident toutes les religions ou préfèrent une religion par rapport à une autre. Ni forcer à ou influencer une personne pour aller ou ne pas aller à l'Église contre sa volonté ni la forcer à professer une croyance ou une non-croyance dans une religion. Aucune personne ne peut être punie pour nourrir ou professer des croyances ou une non-croyance, une pratique religieuse ou une absence de pratique. Aucune taxe d'aucun montant, grand ou petit, ne peut être levée pour supporter des activités ou des institutions religieuses, quel que soit le nom qu'on leur donne [...] Aucun État ni le gouvernement fédéral ne peut, ouvertement ou secrètement, participer aux affaires d'aucune organisation religieuse ou groupe ou vice versa. Dans les mots de Jefferson, la clause contre l'établissement par la loi d'une religion a été décidée pour ériger un mur de séparation entre l'Église et l'État ${ }^{8}$.»

Une autre grande décision fut celle rédigée par le juge Earl Warren, Engel v. Vitale de juin 1962, qui interdit les prières obligatoires dans les écoles publiques au nom du Premier Amendement ${ }^{9}$. L'année suivante, avec les arrêts Schempp v. Abington et Murray v. Curlett ${ }^{10}$, la Cour suprême considère tout type de prière, y compris occasionnelle, ainsi que la lecture de la Bible dans les écoles publiques comme une violation du Premier Amendement ${ }^{11}$, sauf quand elle se fait dans les cours de littérature, d'histoire et d'enseignement comparé des religions. Beaucoup de commentateurs dans le spectre politique et religieux critiquent alors cette décision. Certains s'en offusquent, en rappelant l'affirmation du vénérable juge William O. Douglas dans l'affaire Zorach v. Clauson de 1952: "Nous sommes un peuple religieux dont les institutions présupposent l'existence d'un Être suprême ${ }^{12}$.» Las, la Cour réitère son interprétation en 1971 dans la fameuse affaire Lemon v. Kurztman

en appel et devant la Cour suprême du New Jersey, son affaire est portée devant la Cour suprême qui n'estima pas qu'il y avait eu violation (car les aides au transport étaient versées aux familles et non pas aux écoles) et qui dégagea à l'occasion sa doctrine fondatrice sur la clause.

8. Cette traduction et toutes les suivantes sont des auteurs.

9. Engel v. Vitale, 370 U.S. 421 (1962).

10. Schempp v. Abington Township et Murray v. Curlett, 374 U.S. 203 (1963).

11. Ibid.

12. Zorach v. Clauson, 343 U.S. 306 (1952). 
qui établit trois règles définissant la neutralité étatique : l'action de l'État doit avoir un objectif séculier; ses principaux effets ne visent ni à avantager ni à désavantager une religion; elle ne favorise aucune intrication (entanglement) entre gouvernement et religion. Au début des années 1980, la juge O'Connor développe en opinion complémentaire la doctrine du contrôle d'approbation (endorsement test) qui interdit à l'État toute action qui paraît approuver une religion ${ }^{13}$. En somme, l'État n'a pas de religion, ne professe aucune religion et ne manifeste aucune préférence religieuse...

\subsection{DE LA CLAUSE DE LIBRE EXERCICE À LA PROTECTION DE LA LIBERTÉ RELIGIEUSE}

La deuxième clause du Premier Amendement a fait l'objet d'une reconnaissance tout aussi progressive comme clause de la liberté religieuse. Les contentieux existants au XIX ${ }^{\mathrm{e}}$ siècle concernent la propension des États à interdire de liberté les petits cultes ou des cultes « honnis » ou encore l'agnosticisme. Les adeptes concernés se défendent alors en utilisant cette clause. Le Protestant Establishment, culturel et législatif, s'est substitué pendant longtemps au renoncement aux Églises établies ${ }^{14}$. Les contraintes les plus diverses sont inscrites dans la législation, comme le devoir de prêter serment sur la Bible pour entrer dans la fonction publique, l'interdiction du blasphème passible de peine de mort dans plusieurs États, la lecture obligatoire des Dix Commandements et de la Bible dans les écoles publiques, etc. En 1838, l'État du Massachusetts condamne par exemple un prédicateur devenu panthéiste à soixante jours d'emprisonnement pour avoir « volontairement blasphémé le saint nom de Dieu » en remettant en question la vision biblique de Dieu et la divinité du Christ. L'accusé, Abner Kneeland, accuse la loi anti-blasphème du Massachusetts de violer le Premier Amendement de la Déclaration des droits de l'État, ce que les juges rejettent. Le panthéiste est condamné à de la prison ferme.

Les Blue Laws appelées aussi Sunday Laws - qui instituent le dimanche comme jour chômé consacré au culte et interdit de toute forme de travail, particulièrement commerçant - constituent un autre barrage solide contre les demandes d'exemption, notamment de la part des juifs et des adventistes. De très nombreux litiges finissent devant les juridictions où ils sont tout aussi

13. Lynch v. Donelly, 465 U.S. 668 (1984).

14. Green S. K., The Second Establishment. Church and State in 19th Century America, NY, Oxford University Press, 2010. 
régulièrement rejetés en ce que, selon l'attendu de l'affaire City of Charleston v. Benjamin de 1846, « le jour du Seigneur, le jour de la Résurrection [...] correspond au Sabbat du Seigneur - sa rigoureuse observance dans une communauté chrétienne est celle qui devrait être exigée ${ }^{15} »$.

Avec la consolidation de la diversité religieuse et la poursuite de l'immigration qui multiplie tout autant l'offre que la dissidence religieuse, la fortune de la «liberté religieuse » comme valeur positive et norme judiciaire finit par s'imposer. Elle est l'œuvre de minorités discriminées qui réclament des exemptions aux lois « religieuses » des États, accompagnées dans leur combat par des associations de libres penseurs, et s'organisant pour défendre la «stricte » séparation et obtenir l'abrogation de toutes les « lois bleues». Et le résultat finit par être atteint. On le mesure à deux jurisprudences éloignées dans le temps et pourtant majeures dans l'émergence de la liberté religieuse comme "cœur» de la deuxième clause. L'affaire Reynolds v. United States de $1879^{16}$ et l'affaire Cantwell v. Connecticut de $1940^{17}$.

Reynolds $v$. United States, a permis de définir la liberté religieuse en énonçant les limites de son périmètre. Invoquée par le chef de l'Église mormone pour justifier sa polygamie et celle de son mouvement dans un territoire directement gouverné par l'État fédéral, la liberté religieuse est considérée par la Cour comme absolue et incontrôlable en tant que croyance, mais limitable dans ses conséquences pratiques - en l'occurrence la polygamie - quand elle a comme conséquence de troubler gravement l'ordre civilisé (sic), car aucune exception «ne [pouvait] permettre qu'une doctrine religieuse soit supérieure à la loi du pays, et par conséquent à chaque citoyen de devenir une loi à lui tout seul ».

La jurisprudence Cantwell plus récente - elle date de 1940 - est menée à terme suite à la requête de l'Union américaine pour les libertés civiles. Elle rend caducs les résidus de législations fédérées qui limitaient la liberté des petites minorités, en déclarant inconstitutionnelle la loi d'autorisation du prosélytisme du Connecticut ${ }^{18}$.

15. City of Charleston v. Benjamin, 2 Strob. 508, (S.C. 1846).

16. Reynolds v. United States, 98 U.S. 145 (1878).

17. Cantwell v. Connecticut, 310 U.S. 296 (1940).

18. L'affaire impliquait un Témoin de Jéhovah et la Cour suprême fédérale déclara qu'en vertu des premier et quatorzième amendement, ratifié après la guerre civile en 1878 , interdisant la privation de liberté sans procédure conforme, le défendeur pouvait librement diffuser sa religion. Ce faisant, la Cour suprême incorpore la clause de libre exercice ainsi interprétée dans les obligations des États fédérés. 
Après ces jurisprudences, la Cour suprême a développé une très forte protection de la liberté religieuse personnelle dans les années 1960-1970 et dans des affaires très diverses : dans Sherbert $v$. Verner ${ }^{19}$ en 1963, elle considère comme illégitime de refuser les allocations chômage à une personne adventiste pratiquante qui refuse un emploi comprenant le samedi travaillé. En 1972, elle donne tort à l'État du Wisconsin, dans Wisconsin $v$. Yoder ${ }^{20}$ qui avait incriminé des parents amish refusant d'envoyer leurs enfants à l'école publique après la classe de quatrième. Avec l'affaire Yoder, le contrôle de l'ingérence admissible de l'État en matière de liberté religieuse est redéfini : il ne peut être porté atteinte à la liberté religieuse d'une personne qu'en présence d'un intérêt public impératif et qu'à la condition que l'atteinte en cause soit le moyen le moins lourd pour atteindre l'intérêt impératif en question.

\section{LA POLARISATION POLITIQUE AUTOUR DU PREMIER AMENDEMENT (1970-2010)}

Nous voici arrivés au début des années 1970 à un moment où le panorama que nous décrivons semble se dégager de manière pérenne. Or, c'est justement cette époque qui constitue un temps de bascule assez exceptionnel, au cours duquel la question du lien entre religion, État et liberté entre dans une spirale de polarisation politique entre droite et gauche. Les racines d'une telle polarisation se trouvent dans la révolution sexuelle des années soixante et les mœurs libérales qu'elle a entraînées, dans la sécularisation prononcée du discours public et celle d'une partie de la population américaine, en bref dans l'évanescence relative de l'imprégnation chrétienne propre à la société américaine. Ces phénomènes, complexes et enchevêtrés, ont entraîné une réaction de résistance si vive que le Premier Amendement est devenu un enjeu politique entre droite et gauche américaines.

\subsection{LA NATURE RELIGIEUSE DE L'ÉTHOS AMÉRICAIN DANS LA CLAUSE DE NON-ÉTABLISSEMENT}

Un clivage s'est ainsi instauré autour de la compréhension de la clause de non-établissement, désormais contestée dans sa version "neutraliste ",

19. Sherbert v. Verner, 374 U.S. 398 (1963).

20. Wisconsin v. Yoder, 406 U.S. 205 (1972). 
taxée péjorativement de "séculariste » par ses contempteurs. La neutralité des institutions publiques est-elle si conforme que cela à l'esprit de la clause ? Les institutions doivent-elles être laïques au point qu'aucun lien n'est maintenu avec l'éthos religieux de l'Amérique? Les réponses à ces questions ont engendré une bataille politique, législative et judiciaire plus générale, bataille qui a trouvé son nom : la guerre culturelle.

D'un côté, les associations militant pour une stricte séparation ont investi les médias et les tribunaux pour réagir à des empiétements considérés par elles comme religieux et en contradiction avec la limite du non-établissement. Deux exemples célèbres de cette militance laïque sont à rappeler. L'affaire Newdow, au début des années 2000, du nom d'un activiste athée de Californie qui dénonçait l'inconstitutionnalité du rajout fait en 1954 au serment d'allégeance à la nation par les écoliers américains, lui-même voté en 1942. Le rajout place la nation américaine "sous la protection de Dieu » (under God). Si la cour d'appel du IX Circuit a bien considéré ce rajout comme inconstitutionnel $^{21}$, la Cour suprême a utilisé un argument de procédure pour ne pas juger au fond ${ }^{22}$. L'autre affaire, dite Freedom From Religion Foundation, concerne la plainte d'une célèbre organisation de libres penseurs du Wisconsin qui a contesté en 2008 la constitutionnalité de la "journée nationale de prière », établie par le Congrès en 1952 et fixée en 1988 à la date du $1^{\text {er }}$ mai. Ce jour-là, le président des États-Unis invite officiellement les Américains à se «tourner vers Dieu, en prière et méditation, dans les Églises, en groupe ou individuellement ». En avril 2010, la juge de la US District Court du Wisconsin, Barbara Craab, a jugé cette injonction présidentielle inconstitutionnelle ${ }^{23}$. L'administration Obama a fait appel de ce jugement devant la cour d'appel fédérale du VII ${ }^{e}$ Circuit, considérant l'injonction comme une simple forme de « déisme cérémoniel ». Malgré la mobilisation complémentaire de plusieurs associations, la cour d'appel a donné raison au gouvernement fédéral ${ }^{24}$.

Par ailleurs, la Cour suprême a, dans l'affaire Marsh v. Chambers de $1983^{25}$, défendu le principe que la prière devant le corps législatif n'était pas une violation de la clause, mais une coutume historique. Elle a réitéré cette position concernant les prières au début des conseils municipaux, avec l'arrêt

21. Newdow v. U.S. Congress, 292 F.3d 597 (U.S. 9th Cir. 2002).

22. Elk Grove Unified School District et al. v. Michael A. Newdow et al., 542 U.S. 961 (2004).

23. Freedom From Religion Foundation, Inc v. Obama, 705 F. Supp. 2 d 1039 (W.D.Wis. 2010).

24. Freedom From Religion Foundation, Inc. v. Obama, 641 F.3d 803 (7th Cir. 2011).

25. Marsh v. Chambers, 463 U.S. 783 (1983). 
Town of Greece v. Galloway de $2014^{26}$. Depuis les années 1990, elle semble revenir sur le régime de stricte neutralité scolaire et l'assouplir vers plus d'empathie religieuse. Ainsi, dans les affaires Agostini v. Felton de 1997 et Mitchell v. Helms de $2000^{27}$, elle est revenue sur deux de ses arrêts précédents qui interdisaient tout subventionnement public des écoles confessionnelles. Concernant la mise en place des chèques-éducation délivrés aux parents pour les aider à scolariser leurs enfants, elle a admis que ces chèques pouvaient être attribués, même si les enfants allaient dans des écoles confessionnelles. Plus encore, les organisations confessionnelles - par le biais d'un ensemble de décrets de George Bush Jr, connus sous le nom de Faith based initatives, eux-mêmes décidés pour contrer l'échec d'une loi fédérale de 2000 du même nom - peuvent désormais concourir pour obtenir des financements liés à des programmes sociaux. Le rejet du recours de la Freedom for Religion Fondation contre le directeur de l'Office of Faith Based and Communities Initiatives de la Maison Blanche, mis en place par ces décrets, a confirmé leur légalité28.

Cependant, d'autres résultats ont été plus mitigés pour les défenseurs de l'éthos chrétien de l'Amérique, en matière d'enseignement notamment. La Cour suprême a eu à statuer à plusieurs reprises sur la question de l'enseignement de l'évolution dans les écoles publiques ${ }^{29}$. Elle a approuvé les jurisprudences des cours fédérales des années 1980-1990 qui ne considéraient pas cet enseignement comme une violation de la liberté religieuse des parents, des enseignants ou des enfants. Elle a refusé l'apposition de stickers d'avertissement sur les manuels de biologie ${ }^{30}$ et a rendu caduques toutes les lois dites d'equal treatment dans les États qui admettaient l'enseignement du créationnisme à côté de celui de l'évolution après l'affaire Edwards v. Aguillard de $1987^{31}$. D'autres plaintes sur l'introduction de l'enseignement de l'Intelligent Design ou l'utilisation de stickers le proposant comme une théorie alternative ont été instruites par les cours fédérales dans les années 2000 qui ont toujours statué dans le même sens, défavorable à l'Intelligent Design.

26. Town of Greece v. Galloway, 134 S. Ct. 1811 (2014).

27. Agostini v. Felton, 521 U.S. 203 (1997) ; Mitchell v. Helms, 530 U.S. 793 (2000).

28. Hein v. Freedom From Religion Inc, 551 U.S. 587 (2007).

29. Chelini-Pont B., «Bouter Darwin hors des classes. La saga judiciaire de l'enseignement de l'évolution à l'école et ses répercussions sur la laïcité scolaire aux États-Unis », in Portier Ph., Veuille M. et Willaime J.-P. (dir), Théories de l'évolution et religions, 2011, Paris, Riveneuve, p. 239-250.

30. Freiler v. Tangipahoa Parish School Board of Education, 98-30132 (U.S. 5th Cir. 2000) que la Cour suprême a refusé de revoir.

31. Edwards v. Aguillard, 482 U.S. 578 (1987). 
D'autres requêtes dans ces mêmes années, concernant l'affichage ou la mise en scène des Dix Commandements dans les palais de justice des États qui suggéraient la soumission de la justice américaine à autre chose qu'à la Constitution, ont abouti favorablement en première instance ou ont été tranchées d'une manière fine, à la manière du Conseil d'État français face aux récentes affaires d'exposition de crèches de Noël : pas d'affichage ou de mise en scène permanents à l'intérieur des bâtiments publics, tolérance pour les installations extérieures ou anciennes, comme manifestation d'une tradition culturelle établie ${ }^{32}$.

\subsection{LA DÉFENSE DE LA LIBERTÉ RELIGIEUSE CONTRE LES EMPIÉTEMENTS DE L'ÉTAT}

Quant à la clause de libre exercice et à son interprétation sur la liberté religieuse depuis l'affaire Yoder, la Cour suprême a été accusée de ne pas la respecter, en insistant trop sur l'intérêt impératif des institutions, des services publics ou du gouvernement dans un certain nombre de cas. L'accusation se transforme en rumeur : la liberté religieuse des personnes est en danger face à un État ou une justice aveugle et hostile à ce droit sacré. Ainsi dans United States v. Lee de $1982^{33}$, la Cour avait rejeté la demande d'un fermier amish d'être exempté des cotisations de sécurité sociale pour raisons religieuses, considérant que l'intérêt de l'État était d'éviter les fraudes. De même, dans l'affaire Goldman v. Weinberger de $1986^{34}$, la Cour avait considéré qu'un militaire américain devait porter exclusivement son uniforme, sans ajout de signe religieux ostensible (en l'occurrence une kippa). La jurisprudence Employment Division v. Smith de $1990^{35}$ met le feu aux poudres. Deux employés d'origine indienne licenciés par une clinique pour avoir consommé du peyotl, certes en usage depuis des siècles dans les rituels de leur religion, s'étaient vus refuser les allocations chômage par l'administration compétente de leur État, l'Oregon. La Cour suprême fédérale, sans même faire intervenir le principe

32. Van Orden v. Perry, 545 US 677, 690 (2005); McCreary Co. v. ACLU of Kentucky, 545 U.S. 844 (2005). V. Chelini-Pont B., « Our law comes from God. Les dix commandements devant la Cour suprême des États-Unis », RTDH, n 67, 2006, p. 689-712. Rigal-Cellard B., « La Cour suprême des États-Unis, organe de régulation de la séparation entre les religions et l'État : étude des arrêts sur la présence des Dix Commandements dans les lieux publics », in Singaravelou S. (dir), Laïcité, enjeux et pratiques, Pessac, PUB, 2007, p. 301-320.

33. United States v. Lee, 455 U.S. 252 (1982).

34. Goldman v. Weinberger, 475 U.S. 503 (1986).

35. Employment Division, Department of Human Resources of Oregon v. Smith, 494 U.S. 872 (1990). 
dégagé dans la jurisprudence Yoder, considère le refus comme justifié, les plaignants ayant été renvoyés pour consommation d'un produit hallucinogène interdit par la loi fédérale sur les narcotiques. À partir de cette affaire, une véritable fronde se met en place pour obliger la Cour - et l'ensemble du système législatif - à pratiquer un contrôle strict des lois et situations qui pourraient entrer en conflit avec les convictions religieuses d'un individu.

Une révolution législative se dessine qui transforme les controverses autour de la défense de la liberté religieuse en mouvement politique «pro-religion»: le Religious Freedom Restoration Act (RFRA), loi fédérale sur la restauration de la liberté religieuse votée en 1993, rend obligatoires le contrôle strict de l'intérêt impératif de l'État et l'usage de la solution la moins restrictive. La loi sur la protection de la liberté religieuse et des dons caritatifs, Religious Liberty and Charitable Donation Protection Act de 1998, exclut les dons aux organismes religieux et caritatifs des recouvrements de créances et les intègre dans les dépenses autorisées par les mandataires en cas de faillite. Le Religious Land Use and Institutionalized Persons Act, également de 1998, garantit des protections accrues en faveur des groupes religieux et des personnes en matière foncière (privilèges fiscaux et de crédit, priorités d'achat et de vente) et en matière de respect des croyances religieuses dans le cadre des prisons et des hôpitaux psychiatriques.

Le RFRA, introduit par un démocrate, Chuck Schumer, est adopté à l'unanimité à la Chambre et avec trois votes contre au Sénat, comme une réponse directe à l'affaire Employment Division v. Smith de 1990. Il a provoqué un changement majeur dans l'approche de la Cour suprême des entraves à la liberté de religion. Le cadre juridique qui en résulte offre une protection finalement bien plus forte à la liberté de religion que ne le prévoit la Constitution et cela, au détriment des autres libertés. Désormais, si la Cour conclut qu'une loi impose un «fardeau substantiel » (substantial burden) à la liberté de religion d'un croyant, cette personne peut invoquer l'entrave à sa liberté religieuse pour ne pas y être soumis, à moins que le gouvernement ou le service public fédéral ne démontre que la loi représente le «moyen le moins restrictif » (the least restrictive means) pour la poursuite d'un intérêt impératif. Ce nouveau standard, connu sous l'expression de strict scrutiny est extrêmement difficile à atteindre et rend a priori n'importe quelle loi fédérale vulnérable. Même si en 1997 la loi RFRA a été jugée inconstitutionnelle en cas d'application aux États ${ }^{36}$, elle reste une arme puissante contre toute loi

36. La Cour suprême dans l'affaire City of Boerne v. Flores, 521 U.S. 507 (1997), déclare la loi fédérale RFRA inconstitutionnelle pour les États fédérés, car le $14^{\mathrm{e}}$ amendement fédéral (sur laquelle la loi fédérale repose) ne donne pas au Congrès le pouvoir d'imposer aux 
fédérale en général, dès lors que cette dernière toucherait d'une manière ou d'une autre la liberté religieuse : et c'est ce qui va se passer avec l'Obamacare, surnom de l'Affordable Care Act.

\section{LA BATAILLE CONTEMPORAINE : LA LIBERTÉ RELIGIEUSE COMME DROIT D'EXEMPTION}

C'est ainsi que nous nous trouvons aujourd'hui dans la deuxième moitié des années 2010 avec des tendances qui se creusent et accentuent la polarisation que nous venons de décrire.

\subsection{LIBERTÉ RELIGIEUSE POUR LES ENTREPRISES : UNE BOÎTE DE PANDORE}

Commençons par l'effet contaminant de la décision de la Cour suprême dans l'arrêt Burwell v. Hobby Lobby ${ }^{37}$ de 2014, qui a élargi la protection du RFRA fédéral à une société privée à but lucratif dont les propriétaires se disaient religieusement blessés par une disposition de la loi sur la santé, l'Affordable Care Act (ACA) imposant aux entreprises une assurance maladie incluant la prise en charge de la contraception. Avec pour conséquence d'ouvrir une boîte de Pandore : la liberté religieuse des entreprises ou des sociétés à but lucratif.

En réalité, une telle liberté existe pour les organisations religieuses à but non lucratif, mais l'extension de ces droits aux sociétés à but lucratif permet des douzaines d'autres demandes de dérogations aux lois généralement applicables. En effet, l'arrêt Hobby Lobby n'a pas cherché à définir les conditions strictes qui feraient de l'entreprise une personne morale croyante ou qui encadreraient les conditions de respect des convictions religieuses de ses responsables ${ }^{38}$. La Cour suprême a noté, de façon analogue au jugement

États des modifications dans l'interprétation de cet amendement. Dans l'arrêt de 2006, Gonzales v. O Centro Espirita Beneficente Uniao do Vegetal, la Cour suprême confirme implicitement la validité du RFRA au niveau fédéral. Pour les États, les lois qui peuvent être en conflit avec le Premier Amendement sont jugées en vertu de la norme simple établie par la Cour dans Smith, qui permet au gouvernement de restreindre la liberté religieuse tant qu'il a un but raisonnable et non discriminatoire et qu'il y a une relation rationnelle entre la loi et le but que le gouvernement espère atteindre.

37. Burwell v. Hobby Lobby Stores, Inc., 134 S. Ct. 2751 (2014).

38. Horowitz P., «The Hobby Lobby Moment», Harvard Law Review, Vol. 128, No. 1, 2014, p. 154. 
Citizens United de $2010^{39}$, que les sociétés ne sont que des collections d'individus et que ces groupes d'individus peuvent choisir d'exercer leur liberté religieuse, collectivement et individuellement, tout comme elle avait jugé que ces groupes avaient le droit d'exercer leur liberté d'expression. L'importance de ce précédent et son impact sur l'imagination populaire doivent être rappelés.

Citizens United, société de production cinématographique à but non lucratif fondée par le célèbre homme politique conservateur Newt Gingrich, souhaitait diffuser un film politique à charge sur Hillary Clinton en prévision des primaires démocrates de 2008. Dans sa décision très controversée, la Cour a jugé que les dépenses "politiques » d'une société étaient une forme de la liberté d'expression, digne de protection en vertu du Premier Amendement (sur la liberté d'expression, à la suite des clauses de non-établissement et de liberté de culte). Les sociétés, qu'elles soient à but lucratif ou à but non lucratif, avaient droit à la liberté d'expression. Bien que le jugement n'ait pas établi que les sociétés fussent des personnes, comme certains commentateurs l'ont affirmé, il a néanmoins ouvert le contenu de la personnalité morale des entreprises à des droits constitutionnels réservés normalement aux individus.

Ainsi, en 2014, après avoir déjà jugé que les sociétés avaient droit à la liberté d'expression, la Cour affirme dans l'affaire Hobby Lobby que les sociétés privées à but lucratif ont le droit de gérer leurs affaires suivant les croyances de leurs propriétaires, même si ces croyances les obligent à ne pas se plier aux lois en vigueur. En définitive la question - irrésolue dans l'affaire Hobby Lobby - demeure posée de savoir si les sociétés par actions, en plus des sociétés familiales, peuvent également être en mesure de réclamer des exemptions, en vertu du RFRA. Si tel est le cas, beaucoup craignent que la législation fédérale anti-discrimination, renforcée par les décisions de la Cour Suprême en matière de mariage homosexuel ${ }^{40}$ et par le décret présidentiel sur l'interdiction de la discrimination de genre dans les services fédéraux ${ }^{41}$, puisse être systématiquement contournée par (toutes) les entreprises, en fonction des sentiments religieux des administrateurs ou dirigeants de la société. L'inquiétude est grande du côté des "pro-droits », alors qu'un grand espoir anime le côté des "pro-religion». La vague de cas de sociétés de

39. Citizens United v. Federal Election Commission, 130 S. Ct. 876 (2010).

40. Windsor v. United States, 133 S. Ct. 2675 (2013) déclarant la section 3 du Defense of Marriage Act de 1996 contraire au principe d'égalité devant la loi.

41. Décret présidentiel 13672 du 21 juillet 2014 interdisant la discrimination de genre des employés fédéraux et s'appliquant à toutes les entreprises travaillant avec ces services. 
divers types qui ont entamé des contentieux dans les tribunaux inférieurs semble suggérer que les préoccupations des pro-droits sont bien fondées.

Autre motif de multiplication des plaintes, l'arrêt Hobby Lobby a changé le concept et l'usage du motif de «fardeau substantiel », d'une manière qui élargit encore les types de revendications possibles dans le cadre du RFRA. Avant l'arrêt Hobby Lobby, l'importance du fardeau moral insupportable était une question de droit que le juge devait déterminer. Dans l'affaire Hobby Lobby, la Cour ne cherche pas à examiner l'affirmation du groupe Hobby Lobby selon laquelle la charge morale imposée par l'ACA - payer une couverture maladie incluant la contraception - était substantielle, ce qui laisse entendre que c'est maintenant aux requérants réclamant des droits au nom du RFRA d'apprécier eux-mêmes la lourdeur de ce fardeau moral. Cela élimine la nécessité de démontrer la substance du fardeau et a donc facilité la multiplication des plaintes fondées sur le RFRA. En acceptant l'idée qu'une entreprise puisse être moralement affectée par l'obligation de financer la contraception de ses salariées, l'arrêt Hobby Lobby permet aux employeurs d'imposer leurs croyances à leurs salariés, ce que le juge Ginsburg a d'ailleurs dénoncé dans son opinion dissidente.

\subsection{LA BATAILLE DES MINI-RFRAS DANS LES ÉTATS FÉDÉRÉS}

L'arrêt Hobby Lobby a eu un autre effet d'entraînement. Les défenseurs conservateurs qui brandissent le droit à la liberté religieuse ont été encouragés après cette décision à militer pour obtenir l'adoption de variantes du RFRA au niveau des États - des mini-RFRAs - convaincus que ces lois leur donneraient de solides moyens dans leur lutte contre l'évolution des normes morales liées à la révolution sexuelle, en l'instance le mariage homosexuel civil, indirectement admis par la jurisprudence fédérale Obergefell v. Hodges de $2015^{42}$, ou encore l'avortement précoce, encadré par plus de 500 lois dans les États.

Depuis l'arrêt Hobby Lobby, 22 États ont ainsi introduit ou mis en chantier la modification de leur loi RFRA, dont certaines incluent explicitement les sociétés, comme l'Oklahoma, quand les autres conservent l'interprétation judiciaire fédérale de l'arrêt Employment Division v. Smith. Il convient de

42. La Cour devait répondre de la plainte de quatorze citoyens contre les lois de plusieurs États américains sur le mariage qui réaffirmaient son caractère hétérosexuel. Les plaignants considéraient que ces lois constituaient une violation du $14^{\mathrm{e}}$ amendement fédéral. Et la Cour suprême leur a donné raison par cinq voix contre quatre. 
noter cependant que ces récentes tentatives de durcir la protection de la liberté religieuse par les mini-RFRAs ont été âprement combattues. À cette date, seuls l'Indiana, l'Arkansas et la Virginie ont réussi à adopter une loi ou des amendements proches du RFRA - signé en Indiana par le gouverneur de l'État, Mike Pence - et ils ont eu à faire face à une résistance très vive. Des manifestants ont tenté en mars 2015 de s'opposer au passage de l'Indiana Religious Freedom Restoration Act, faisant écho à des manifestations similaires en Géorgie, dans l'Arkansas, le Colorado ou encore le Michigan, au nom de la prévisible discrimination dont pourraient être victimes les homosexuels. En effet ces États - et ils sont majoritaires - n'ont pas de disposition qui interdise la discrimination pour orientation sexuelle. Si l'Indiana a finalement inclus une clause interdisant aux entreprises de refuser leurs services ou leurs biens à un citoyen en vertu de leurs convictions religieuses blessées, d'autres législatures comme celle de l'Oklahoma ont des projets de textes en discussion qui autorisent directement des individus et/ou des " entités religieuses» qui peuvent être des entreprises privées, à avoir un droit de réserve religieuse, ce qui signifie concrètement qu'ils peuvent refuser leur service à des clients, refuser d'embaucher du personnel ou licencier pour raison religieuse ${ }^{43}$.

\subsection{DES PLAINTES FONDÉES SUR L'IDÉE DE REFUS DE COMPLICITÉ}

Corrélativement à cette multiplication des mini-RFRAs, nous assistons à une montée des plaintes pour refus de complicitét ${ }^{44}$. Il s'agit d'une catégorie de revendications faites par des individus ou des groupes qui refusent de se conformer aux lois communes, non parce qu'elles les empêchent d'exercer une quelconque obligation religieuse positive, mais parce que cette adhésion les rendrait complices des péchés des autres.

43. Le texte en discussion intitulé Oklahoma Religious Freedom Restoration Act de 2015 (OK SB 440 2016) élargit la définition de l'organisation religieuse, exemptée au nom de ses croyances concernant « le sexe, le genre ou la sexualité " d'admettre la validité légale d'un mariage homosexuel ou de procurer ses services ou ses offres d'emplois à des couples homosexuels. L'organisation religieuse redéfinie inclut les entreprises privées « opérant de manière cohérente avec des convictions religieuses sincères ». De nouveaux amendements au projet ont été introduits en 2016 : précision de ce que l'intérêt vital de l'État ne peut jamais permettre de forcer quelqu'un à participer à une cérémonie de mariage (homosexuel) ou à fournir ses services en vue de ce genre d'activités (OK HB1371 2016); précision de ce que l'exercice de la religion inclut dans sa définition la faculté d'agir ou de refuser d'agir en accord avec sa foi (OK SB 898 2016).

44. NeJaime D. \& Siegel R. B., « Conscience Wars: Complicity-Based Conscience Claims in Religion and Politics », Yale Law Journal, Vol. 124, 2015, p. 2516-2591. 
L'idée elle-même n'est pas nouvelle aux États-Unis, ni propre d'ailleurs à la culture américaine: dans les années 1980, le mouvement de lutte contre la ségrégation et les décisions de sanctions contre l'Afrique du Sud sont un exemple fort de l'argument de refus de complicité, qui a recueilli un soutien très large aux États-Unis et dans la communauté internationale. Cependant, les cas qui se sont posés récemment sont fondés sur l'idée qu'on ne doit pas être complice du péché d'autrui. Cette idée, couplée avec l'approche rigoureuse du RFRA en faveur de l'accommodement religieux, est une autre arme puissante pour les conservateurs religieux, cherchant les biais de résistance aux évolutions sociétales libéralisant la sexualité.

Le succès de l'arrêt Hobby Lobby a révélé - nous l'avons vu - une modification dans l'approche de la Cour concernant l'évaluation du fardeau dit substantiel. La Cour ne s'est pas jugée compétente pour décider si le lien entre les actions requises par la loi et le "péché » induit par ces actions, dont le plaignant ne veut pas être complice, est trop ténu pour être pris au sérieux. Dans la doctrine majoritaire de la Cour, le contenu subjectif de l'exercice de la religion - comme absolu du for interne extérieurement limitable selon la jurisprudence fondatrice du cas Reynolds - est devenu à la fois insaisissable et prioritaire.

Dans l'affaire Hobby Lobby, ces droits impliquaient l'accès à la contraception en vertu d'un contrat d'assurance fourni par l'employeur, comme le prescrit l'ACA. Mais dans les tribunaux inférieurs, une autre sorte de cas a attiré l'attention du public, davantage focalisée sur la légitimité religieuse à discriminer les homosexuels, malgré les lois et jurisprudences qui entérinent leur existence sociale. Le cas Elane Photography ${ }^{45}$ de 2013 concerne un photographe professionnel qui a refusé de photographier une célébration de mariage homosexuel, au motif de sa désapprobation spirituelle. Le couple lésé a porté plainte en vertu de la loi anti-discrimination du Nouveau-Mexique - où se situait l'affaire - qui interdit expressément aux entreprises de pratiquer une discrimination fondée sur l'orientation sexuelle. La défense du photographe s'est basée sur son refus d'être complice du péché que représente le mariage homosexuel. La Cour suprême du Nouveau-Mexique s'est finalement opposée aux demandeurs, jugeant que le RFRA du Nouveau-Mexique ne s'appliquait pas, puisque le photographe n'était pas une entreprise, et qu'il s'agissait d'un simple conflit entre parties privées. Autre exemple, en décembre 2016, une cour d'appel fédérale du Texas a prononcé une injonction préliminaire, en citant le RFRA, contre un décret gouvernemental d'application de l'ACA

45. Elane Photography, LLC v. Willock, 309 P.3d 53 (NM 2013). 
qui interprète la discrimination de genre comme une discrimination sur la base de l'identité sexuelle. Il semble que les débats sont loin d'être terminés.

\subsection{CONTESTATIONS SOCIÉTALES AU NOM DE LA LIBERTÉ RELIGIEUSE}

La lutte en faveur de la liberté religieuse des entreprises et la montée des revendications de refus de complicité semblent avoir en commun la nouvelle orientation qui est donnée à la liberté religieuse, en faveur de contestations sociales conservatrices contre l'évolution des normes admises, principalement en matière de santé reproductive, de vie de couple et de relations sexuelles. Quand bien même l'emprise des normes morales chrétiennes sur la vie des gens a singulièrement diminué aux États-Unis sous l'effet de la sécularisation d'après-guerre et de l'extension du libéralisme aux comportements sexuels, les conservateurs américains ont construit leur idéologie politique sur le rétablissement d'une République chrétienne unie et mythifiée qui inclut le maintien ou la réorganisation d'un droit de la famille en conséquence. Ils rejettent formellement l'idée que le libéralisme sociétal fasse partie de l'histoire et de la culture américaines des droits et ils ne cherchent pas non plus à user d'arguments philosophiques pour affirmer l'existence de principes moraux universels à la source de la loi commune qui s'imposent à chacun. Leur langage est bien celui du rappel de l'autorité divine injonctive à laquelle une population chrétienne se doit d'être conforme. Les conservateurs ont donc investi la liberté religieuse comme une autre arme politique, après avoir dénoncé la dérive séculariste forcée de la séparation américaine, alors que celle-ci, sur le long terme, paraît s'être adaptée depuis l'époque répressive du Protestant Establishment aux besoins d'une société chrétienne pluraliste, puis à ceux d'une société pluraliste avec une majorité chrétienne aujourd'hui partiellement sécularisée et comprenant un quart de personnes se déclarant sans religion. Face à cette évolution, le « combat » pour la liberté religieuse défend autre chose qu'un contenu classique bien défini de droit universel de croire et de pratiquer. La liberté religieuse est passée chez ses défenseurs d'un récit paradigmatique de protection de la minorité à celui de protection de la majorité croyante.

C'est exactement l'enjeu des cas de refus de complicité face au mariage homosexuel. Avec la légalisation du mariage homosexuel dans une quinzaine d'États, des cas de refus de complicité ont commencé à se multiplier, dans lesquels les entreprises ou artisans refusaient de fournir des services aux couples homosexuels, arguant qu'ils avaient le droit de discriminer pour 
ne pas être complices de leur péché, comme dans le cas Elane Photography. Une autre affaire a impliqué un fleuriste, et une autre encore un boulanger chrétien qui a refusé de créer un gâteau de mariage pour un couple de même sexe $^{46}$. Certains cas sont presque drôles : un client chrétien a commandé à une boulangerie gay-friendly de préparer des gâteaux avec des messages tels que «l'homosexualité est un péché détestable ${ }^{47}$ ». Les tribunaux ont soutenu jusqu'à présent le droit du gouvernement à adopter et à faire respecter les lois antidiscriminatoires. Au niveau fédéral, les décisions de la Cour suprême de 2013 et 2015 sur des cas relatifs au mariage gay ont utilisé - nous l'avons $\mathrm{vu}-\mathrm{le} 14^{\mathrm{e}}$ Amendement et donné raison aux plaignants. Le passage à des RFRAs plus stricts encore, comme celui de l'Indiana ou le projet de l'Oklahoma, semble donc très difficile. La société américaine s'est orientée vers l'acceptation de l'homosexualité et ni les tribunaux ni les législateurs - ni la plupart des entreprises, d'ailleurs - n'ont d'intérêt réel à promouvoir une discrimination légalisée et spécifique la concernant.

Outre les cas relatifs au mariage homosexuel, nous avons vu aussi que la plupart des plaintes fondées sur le refus de complicité ont concerné l'accès aux services contraceptifs dans le cadre de l'ACA. Ces revendications ont rencontré un plus grand succès, comme l'a démontré l'affaire Hobby Lobby. Dans plusieurs affaires récentes ${ }^{48}$, les plaignants ont rejeté le compromis proposé par l'arrêt Hobby Lobby : être autorisés, comme c'était déjà le cas pour les organisations religieuses, à déposer un formulaire notifiant l'objection de l'assureur au paiement de la couverture contraceptive. Ces plaignants ont insisté sur le fait qu'une telle notification les rendrait complices, puisque l'avis «déclencherait» la contraception par d'autres moyens! Bien que les tribunaux inférieurs aient rejeté ces allégations, la Cour suprême a récemment renvoyé plusieurs cas similaires devant ces tribunaux, afin d'examiner les éventuels compromis, suggérés dans des mémoires supplémentaires ${ }^{49}$.

46. State of Washington v. Arlene's Flowers, 187 Wn.2d 804 (2017) ; Craig v. Masterpiece Cakeshop, Inc, 2015 COA 115.

47. Jack v. Azucar Bakery, Colorado Civil Rights Commission Determination, Charge No. P20140069x (2015).

48. Par ex., Little Sisters of the Poor Home for the Aged v. Burwell, No. 13-1540 (10th Cir. 2015); Wheaton College v. Burwell, 791 F.3d 792 (7th Cir. 2015).

49. Zubik v. Burwell, 136 S. Ct. 1557 (2016). 


\section{PROSPECTIVE SOUS LE MANDAT DU PRÉSIDENT DONALD TRUMP}

La plupart des litiges récents concernant la liberté de religion se sont donc concentrés sur des causes idéologiques «de droite », en particulier le droit à la discrimination et le droit de ne pas fournir une assurance maladie obligatoire incluant le mandat contraceptif. Il paraît certain que sous la présidence Trump, le mandat de contraception de l'ACA sera supprimé, si ce n'est l'ACA lui-même.

En ce qui concerne la discrimination à l'égard du comportement homosexuel, même si Donald Trump a déclaré dès janvier 2017 ne pas vouloir revenir sur le décret présidentiel de Barack Obama interdisant la discrimination de genre pour les employés fédéraux, le nouveau président a aussi promis, aux premiers jours de son mandat, de signer une loi proposée en 2015 et qui pourrait être votée cette année par le $115^{\mathrm{e}}$ Congrès. Le First Amendment Defense Act retirerait au gouvernement fédéral le droit d'empêcher les personnes d'affirmer au nom de leur conviction religieuse qu'on ne doit reconnaître que les mariages entre un homme et une femme et/ou qu'on ne doit pas avoir de relations sexuelles hors d'un tel mariage. Si elle entre en vigueur, cette loi fédérale autoriserait "globalement » la discrimination contre les couples homosexuels ou les couples non mariés de la part de personnes se considérant comme religieuses, y compris les sociétés, au nom de leur liberté religieuse nouvellement admise.

Les groupes conservateurs sont donc susceptibles de renouveler leurs efforts pour limiter, supprimer voire transformer la législation fédérale et les dispositions existant dans les États contre la discrimination sexuelle, à travers des cas-tests soigneusement mis en scène. Toutes ces affaires prendront de plus en plus d'importance avec la nomination du nouveau juge de la Cour suprême pour remplacer Antonin Scalia, décédé en mai 2016. Depuis la mort de ce dernier et le refus du Sénat républicain d'auditionner le candidat proposé par Barack Obama, les dossiers les plus sensibles ont été laissés en attente. Avec un nouveau juge conservateur, Neil Gorsuch, proposé par Donald Trump au $1^{\text {er }}$ février et accepté le 3 avril 2017 par la Commission de la justice du Sénat, la Cour sera probablement plus accommodante dans les cas de refus de complicité, mais il est peu probable qu'elle utilisera le RFRA pour permettre un droit à la discrimination. Cependant, si un juge de plus prenait sa retraite ou mourrait pendant la présidence Trump, une deuxième nomination conservatrice ouvrirait la porte à une majorité de juges plus enclins à interpréter la clause de libre exercice en libre exercice de la conscience chrétienne. 
Enfin, en matière de non-établissement, deux autres actions du nouveau président promettent de susciter des résistances et possiblement de nouveaux conflits juridiques. En signant ses deux décrets présidentiels sur l'interdiction temporaire ou sine die d'entrée sur le territoire de citoyens de sept puis six pays musulmans « à risque » et le blocage également temporaire de l'admission des réfugiés - décrets immédiatement retoqués par la justice les 3 février et 6 mars $2017^{50}$ - le nouveau président a promis que les réfugiés chrétiens syriens seraient ensuite préférés aux musulmans. Une telle politique serait une violation directe de la clause de non-établissement et probablement de la clause de protection égale, contenue dans le $14^{\mathrm{e}}$ Amendement, en ce qu'elle favorise une religion spécifique. La deuxième action, promise par Trump à ses soutiens religieux, a également été appliquée, celle d'abroger le Johnson Amendment, clause du Code fiscal qui empêche les Églises de militer pour ou contre des candidats politiques ${ }^{51}$. Cela ouvre la possibilité d'une influence politique directe des Églises et d'un potentiel détournement de la mission des Églises par l'argent politique. Étant donné que ces deux craintes étaient précisément celles invoquées par James Madison pendant la rédaction de la clause d'établissement en 1789 , on peut s'attendre, sur ces questions aussi, à une période de conflits importants au cours des prochaines années. On le voit, la bataille du Premier Amendement n'est pas terminée, pas plus que la guerre culturelle des deux Amériques.

50. State of Washington and State of Minnesota v. Trump, No. 2: 17-cv-00141 (W.D. Wash. 2017) ; State of Washington et al. v. Trump, No. 17-35105 (U.S. 9th Cir. 2017) ; State of Hawaii v. Trump, No. 17-15589 (U.S. 9th Cir. 2017).

51. Presidential Executive Order Promoting Free Speech and Religious Liberty, 4 mai 2017. 